- Palabras clave/ Ciudad-paisaje, megaestructuras, arquitectura escalonada, helioterapia.

$\Delta$ Keywords/ landscape-city, mega-structures, stepped-back architecture, heliotherapy.

$\Delta$ Recepción/ 13 octubre 2017

A Aceptación/ 14 febrero 2018

\section{La arquitectura escalonada como generatriz de la ciudad-paisaje $(1907-1964)^{1}$}

\author{
The stepped-back architecture: The matrix of \\ the landscape-city (1907-1964)1
}

\section{Eduardo Jiménez-Morales}

Arquitecto, Universidad de Granada, España Doctor en Urbanismo y Ordenación del Territorio, Universidad de Málaga, España. Docente e investigador, Departamento de Arte y Arquitectura, Universidad de Málaga, España. eduardo_jmauma.es

\section{Ingrid Vargas-Díaz}

Arquitecta, Universidad de Ibagué, Colombia: Universidad de Granada (UGR), España. Doctora en Urbanismo y Ordenación del Territorio, Universidad de Granada, España. Académica e investigadora, Clúster de Investigación N-URB, España. ingrill@hotmail.com

RESUMEN/ El propósito de este artículo es constatar la importancia que tuvo la arquitectura escalonada en la conceptualización de la ciudad-paisaje en la década de 1960. Especialmente, porque fue su sección de terrazas retranqueadas la que se utilizó de manera recurrente como generatriz para dar forma a las mega-estructuras, sin duda, las propuestas más innovadoras de la época. Para ello se analizan las relaciones que se establecen entre esta vocación formal y la arquitectura de sección escalonada que se desarrolló en el período de entreguerras. Esta última respondió de ese modo a las inquietudes suscitadas sobre la salud pública en la planificación de la ciudad o en el trabajo, y encontró en la arquitectura hospitalaria sus mejores referentes. Este fue el caso del Freilufthaus, resultado de la adecuación formal de la arquitectura del sanatorio antituberculoso a las exigencias de la helioterapia al inicio del siglo XX. ABSTRACT/ This article is aimed at establishing the importance of stepped-back architecture in the conceptualization of the landscape-city during the sixties. Particularly so, since its setback terrace section was persistently used as the matrix to shape mega-structures, undoubtedly the most innovating proposals for the epoch. The article discusses the relationships between this formal vocation and the stepped-back architecture that developed during the interwar period. This was architecture's way to deal with the concerns regarding public health in the planning of cities and work, and found its best models in hospital architecture. This was the case of the Freilufthaus which resulted from a formal adaptation of the tuberculosis sanitarium to the requirements of $20^{\text {th }}$ century heliotherapy.

INTRODUCCIÓN. En los inicios de la segunda mitad del siglo XX, la idoneidad de los postulados del Movimiento Moderno y del Estilo Internacional sobre el modelo de ciudad comenzaron a ponerse en cuestión Dichos cuestionamientos fueron planteados, particularmente, por una generación de arquitectos que emprendieron entonces la búsqueda de nuevas estrategias afines a la idea de ciudad que emanaba de las revoluciones sociales, puestas en marcha durante la década de 1960. Sus propuestas, teóricas en muchos casos, se convertirían en herramientas críticas frente a la ortodoxia moderna, considerada insatisfactoria y alienante, a la vez que reivindicarían el papel activo que debe jugar el individuo en el proceso de construcción y de transformación de la ciudad (Habraken 1961).

En los libros Les cités de l'avenir (1966) y Où vivrons-nous demain? (1963) del escritor Michel Ragon, se exploró el estado de la ciudad moderna y sus posibles futuros, mientras que el artista John McHale recogía visiones insólitas sobre la arquitectura en The Future of the Future (1969) y en el número AD 2000+ (1967) de Architectural Design. Asimismo, los libros de predicciones arquitectónicas Architecture: Action and plan (1967) y Experimental Architecture (1970), obras de Peter Cook; y los catálogos sobre estructuras urbanas Stadtstrukturen für morgen (1971) de Justus Dahinden y Megastructure. Urban futures of the recent past (1976) de Reyner Banham, entre otros, completaban la avanzada en busca de ese otro futuro para la ciudad.

No obstante, fue este último, Reyner Banham y su compendio históricocrítico sobre las mega-estructuras, el que puso el foco sobre las propuestas que habían canalizado una gran parte de los conceptos e innovaciones formales que surgieron a lo largo de esos años, muchas de las cuales eran dominadas por agrupaciones de unidades mínimas, intercambiables y asociadas a un sistema

Esta investigación ha sido posible gracias al Programa UMA FC 2015-004 para el Fortalecimiento de Recursos Humanos en Investigación de la Universidad de Málaga. Su arco temporal lo define la presentación del Freilufthaus en el XIV Congreso Internacional de Higiene y Demografía (1907) y el que fue considerado por Reyner Banham como el Mega-año (1964). 
estructural de soporte, estable y, al mismo tiempo, adaptable a las variaciones de sus componentes. Su resultado formal estuvo condicionado a cómo se ensamblaban estos elementos. Sin embargo, de manera recurrente, se utilizó la sección de terrazas escalonadas como generatriz con la que modelar la mega-estructura como una geografía vertical habitable (imagen 1) Las propuestas para Villes cratères (19631969) de Chanéac, Intrapolis (1958) de Walter Jonas o Hill City (1968-1972) de Justus Dahinden son buenos ejemplos de ello. Cabe también mencionar otros ejemplos, como el Sunset Mountain (1964) de César Pelli y Anthony Lumsden, o la propuesta Crater City (1971) de Peter Cook Las primeras, abordaban la relación del hombre con su entorno artificial a partir de la reinterpretación de las formas de la naturaleza en la ciudad. Las segundas, hacían uso de la orografía natural como soporte de sus proyectos. Sin embargo, todas coincidían en la crítica a la práctica moderna y en la preocupación por la continuidad y el contexto, introducidas con la idea de ciudad-paisaje en el pensamiento urbanístico de los años sesenta. Fumihiko Maki reconoció en estas estrategias similitudes con "(...) esa gran colina sobre la que se construyeron las ciudades italianas" (Maki 1964: 8), entroncándolas así con las experiencias de Schinkel, Hoffmann o Le Corbusier durante sus viajes por el Mediterráneo. No obstante, el propio Banham o Dahinden reconocían también el potencial de la arquitectura escalonada para asegurar el soleamiento óptimo de las viviendas, muy evidente en las propuestas de Ecopolis (1972) o Solar Village (1975), obras de Guy Rottier y James Lambeth, respectivamente. A pesar de ello, las referencias al origen de la arquitectura solar como generatriz de la ciudad-paisaje han sido escasas y, por ello, son objeto de estudio a lo largo del presente artículo. Más allá de la semejanza formal con la arquitectura vernácula mediterránea, convertida por la disciplina arquitectónica en referente histórico de las mega-
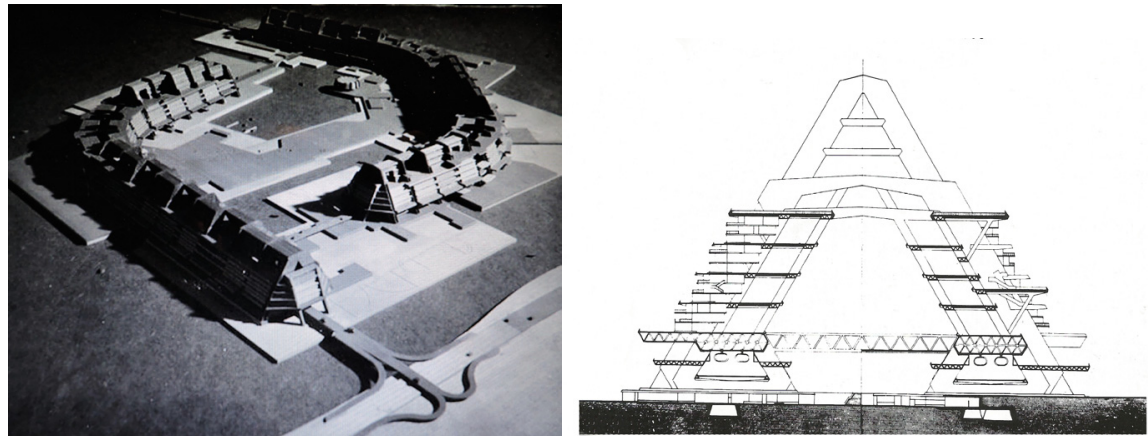

Imagen 1. Community for 25,000 de Kenzo Tange. Sección transversal (fuente: Nyilas 2006: 182)
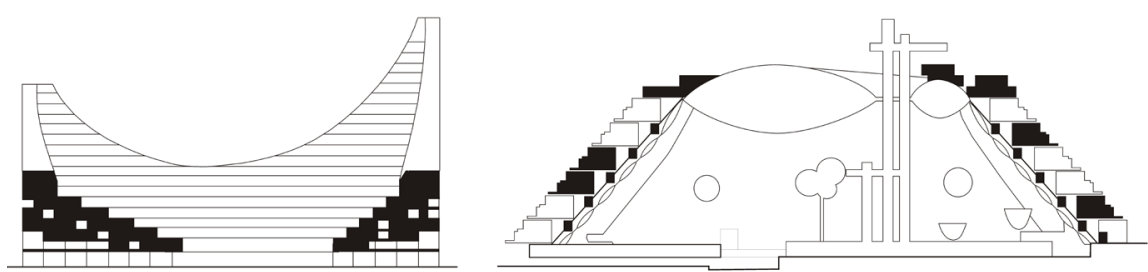

A

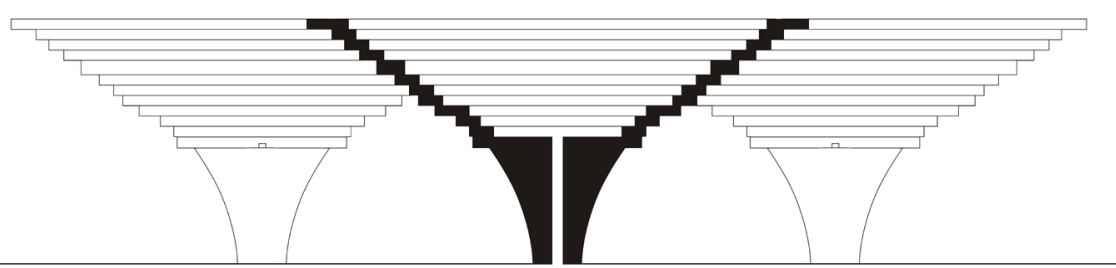

C

Imagen 2. Sección transversal a las propuestas para Villes Cratères (A), Hill City (B) e Intrapolis (C) de Chanéac, Justus Dahinden y Walter Jonas, respectivamente (fuente: Elaboración propia).

la ciudad aterrazada que se promovió en el libro Terrassen-typ (1929) de Richard Döcker, donde el edificio-terraza -paradigma de ese modelo de habitar- garantizaba el acceso a unas condiciones saludables de luz y ventilación en la ciudad. Su naturaleza sanitaria parece, además, entroncarlo con algunas de las propuestas arquitectónicas hospitalarias que aportaron los médicos al inicio del siglo XX, sobre todo con aquellas destinadas a implementar la helioterapia como tratamiento contra la tuberculosis. Para comprobar esta hipótesis, el artículo se estructura en cuatro apartados. El primero aborda la experimentación arquitectónica en torno al edificio-terraza como respuesta a la necesidad de salud ambiental en la planificación de la ciudad. El segundo, aporta los referentes que utilizaron los arquitectos de la época para realizar estas indagaciones, muchos de los cuales se relacionaron con una de las soluciones arquitectónicas aportadas por los sanatorios antituberculosos a comienzos del siglo XX: el Freilufthaus. El tercer apartado expone el origen de este modelo aterrazado como resultado del progreso de la arquitectura bajo el dictado de las exigencias médicas. Finalmente, el último apartado recoge las principales conclusiones. 
dentro del contexto parisino. Frente a las inquietudes suscitadas sobre la salud pública en la ciudad y en el trabajo desarrolló primero immeubles à gradins como una respuesta higienista para las viviendas obreras. Luego, ensayó con su escala, haciendo extensible su arquitectura a toda la ciudad. Sus propuestas utópicas para Le Giant Hotel (1927) o Métropolis (1928) dan muestra de ello, en especial esta última, manifiesto futurista sobre un edificio-ciudad de terrazas retranqueadas, cuya autonomía funcional y monumentalidad rompió con los esquemas post-haussmanianos de su época (imagen 4).

Adolf Loos ya le había otorgado credibilidad a esta idea en 1923, cuando expuso su propuesta del Grand Hotel Babylon (imagen 5) en el Salon d'automne $e^{2}$ parisiense. Loos había iniciado esta reflexión con sus prototipos de vivienda colectiva en Viena, donde terrazas escalonadas, a modo de calles elevadas, permitían aunar la vida en la ciudad con el ideal perdido de la casa como espacio habitable. El proceso de individualización del espacio terraza como prolongación natural del hábitat en el exterior culminó con su proyecto de Veinte Villas (1923) y con el propio Grand Hotel Babylon, cuyo esquema compositivo piramidal aseguraba la máxima exposición al sol y una proporcionada inserción del edificio en el paisaje urbano.

En cualquier caso, tanto la disolución de límites entre el exterior y el interior, la adecuada orientación de los espacios y la consecuente diferenciación de las fachadas, así como la frontal oposición a las estancias mal ventiladas en favor de su apertura a aire y al sol, entre otros, son rasgos que se repiten en cada una de estas propuestas. Se trata siempre del resultado de una incesante búsqueda de la salud ambiental en la ciudad a través de la experimentación con la vivienda colectiva. Así, muchos arquitectos recurrieron a las soluciones que les ofrecía la arquitectura hospitalaria, ya que, a comienzos del siglo XX, fueron los médicos los primeros en apostar por el recurso de la sección escalonada como modelo para el hábitat colectivo.

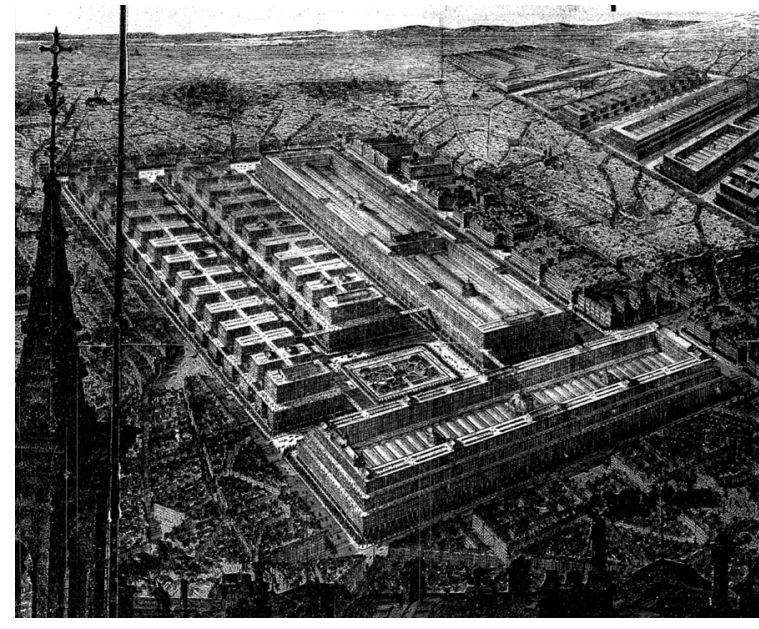

Imagen 3. Aérodômes, propuesta para los suburbios de París (fuente: Borie 1865: 47).

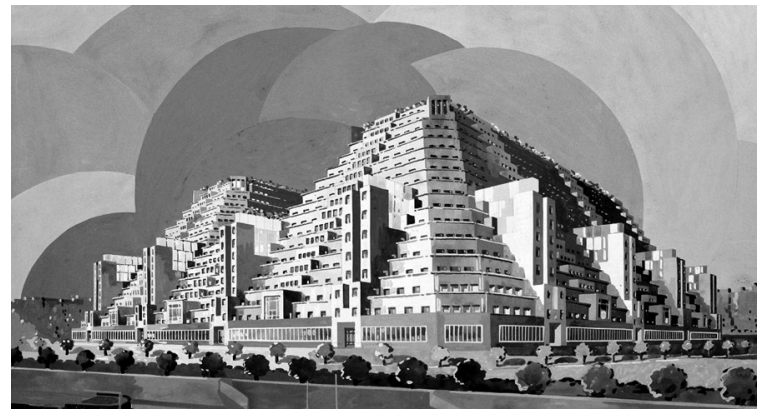

Imagen 4. Edificio Métropolis, quai de Passy, París 8 e ffuente: Sauvage s/f.)

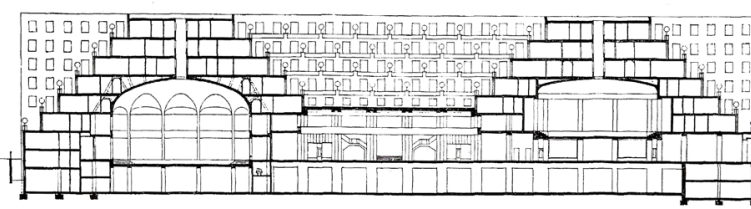

Imagen 5. Sección longitudina/ de/ Grand Hotel Babylon en Niza (fuente: Loos 1928: 337). puentes peatonales. Su seccion y bien aireados para cada nivel, a la vez que modificaba el perfil tipo de las calles

Pero fue Henri Sauvage quien explotó de manera sistemática las posibilidades ofrecidas por la arquitectura escalonada 
DEL SANATORIO AL TERRASSENBAU.

Durante el período de entreguerras,

algunos médicos y arquitectos europeos

se concentraron en analizar, de una manera

crítica, las soluciones aportadas por la

arquitectura hospitalaria desde 1900,

evaluando, a su vez, su posible transferencia

a otros programas, tales como el educativo

o el residencial. Su objetivo era recopilar

aquellos proyectos evocadores de una

nueva cultura del habitar, capaces de aunar

el acceso al aire y la luz con una especial

atención a la higiene y a la salubridad. Libros

como Neuzeitliche Hotels und Kranken-

Häuser (1929) del alemán Hermann Gescheit, Hotels et Sanatoria (1930) del francés

Gabriel Guevrekian o Befreites Wohnen

(1929) del suizo Sigfried Giedion, son buena muestra de ello.

Pero fue el médico alemán Julius Grober quien puso énfasis en los modelos

sanatoriales que se habían diseñado según

el esquema de terrazas escalonadas hasta la

fecha. En su libro Das deutsche Krankenhaus

(1932), Grober destaca, además, la variante

que aportaba el arquitecto alemán Richard

Döcker en el Waiblingen County Hospital de 1928 (imagen 6). Se trataba de un doble juego de retranqueos y voladizos que subsanaban la falta de luz y de ventilación de los espacios en sus niveles inferiores, gracias al doble escalonamiento de su fachada principal y su trasera. Su sección escalonada también aparecería en Terrassen-typ (1929), la publicación de Döcker que recogía la esencia de este proyecto: "(...) lo que hace verdaderamente saludable a esta clínica es el uso de sus terrazas, ley ampliable a cualquier tipología, ya sea vivienda colectiva o unifamiliar, hotel o edificio de oficinas. Con ello se puede garantizar incluso la salubridad de la ciudad y, por extensión, (...) embellecer su torturado perfil" (Döcker 1929: 1)

Esta generalización del Terrassenbau o edificio-terraza como paradigma de una nueva forma de habitar la ciudad, ya había sido abordada por el doctor David Sarason, cuando se propuso trasladar la arquitectura del sanatorio escalonado a la residencia colectiva en su libro Ein Neues Bauprinzip für Krankenanstalten und Wohnhäuser (1913). Sin embargo, fue la revista L'Architecture (1902) la que publicó primero esta idea, tras hacerse eco del interés del doctor Camille Savoire por los modelos sanatoriales alemanes, sobre todo por el Freilufthaus, un edificio sanitario de terrazas retranqueadas elaborado por el propio Sarason hacia 1900 y que, según la historiadora Marie J. Dumont (1984), Henri Sauvage ya conocía cuando diseñó los immeubles à gradins.

Richard Döcker también publicó en su libro Terrassen-typ el diseño original del Freilufthaus con la intención de comparar su sección transversal con la del Waiblingen County Hospital y la de uno de sus más reconocidos predecesores: el Sanatorio Franco-Americano del arquitecto Tony Garnier; y evaluar, así, sus respectivas calidades arquitectónicas a partir de las proporciones del vuelo de sus terrazas, o de la altura libre y profundidad de sus habitaciones con respecto a la inclinación solar (imagen 7). Aunque es indudable el rigor médico con el que se concibieron cada uno de estos proyectos, el modelo de Sarason ejemplificó, como ningún otro, el progreso de la arquitectura bajo el dictamen de las necesidades médicas del momento.

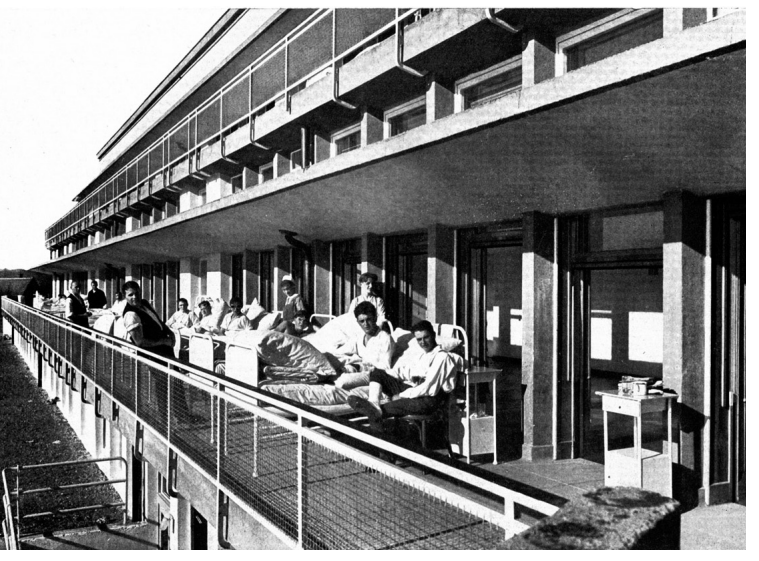

Imagen 6. Pacientes en la terraza de/ Waiblingen County Imagen 6. Pacientes en la terraza
Hospital (fuente: Döcker 1929: 13).

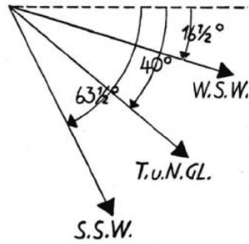

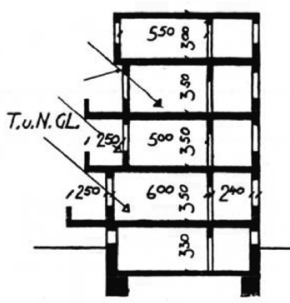

A

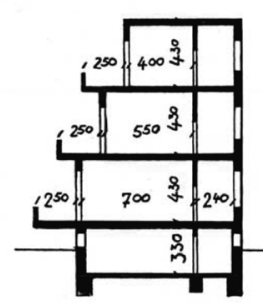

B

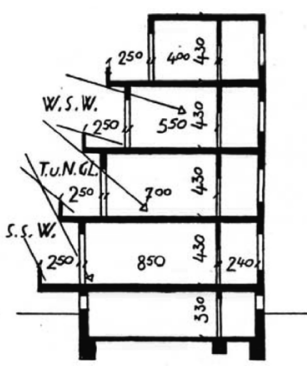

C
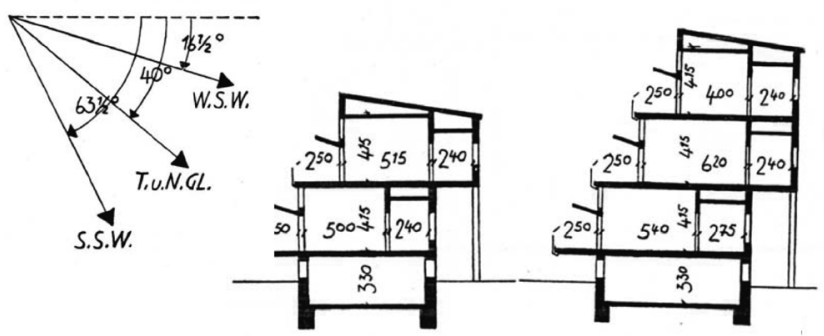

$\mathrm{F}$
G

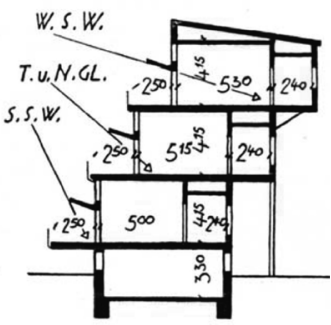

$\mathrm{H}$
Imagen 7. Estudio comparativo de la incidencia sola Freilufthaus $(A$ y B ) (fuente: Döcker 1929: 62-63). 
LA HELIOTERAPIA Y EL

FREILUFTHAUS. Al finalizar el siglo XIX en un mundo que se había industrializado rápidamente, la confianza del cuerpo médico en las propiedades salutíferas de la naturaleza favoreció un nuevo impulso en la formalización arquitectónica del sanatorio. La incorporación de la terapia solar para hacer frente a algunos cuadros de tuberculosis, se acompañó de cubiertas planas, solariums y fachadas escalonadas que fueron reemplazando a las galerías de cura cubiertas, tan representativas de los sanatorios desde 1876 (Jiménez y Vargas 2017). Se aseguraba así que el enfermo pudiera exponerse al sol desde su habitación, siguiendo las pautas establecidas por los doctores Oskar Bernhard o Auguste Rollier, promotores de la helioterapia desde 1902
No obstante, el médico alemán David Sarason y el arquitecto berlinés Gustav Bähr, fueron los responsables de idear el sanatorio escalonado o Freilufthaus hacia 1900 (Sarason 1913). Su propuesta constaba de cuatro niveles de habitaciones cuyo frente sur se retranqueaba en altura para dar forma a terrazas semicubiertas, expuestas al aire y al sol, frente a los amplios ventanales de cada una de ellas (imagen 8). Entre 1908 y 1910, el arquitecto Henry Verrey solo consiguió reproducirlo parcialmente para el Children Sanatorium de Lausana (Suiza), dado que la posibilidad de ejecutarlo en su totalidad iba a quedar relegada a la incorporación del hormigón armado como material de construcción estructural.

A pesar de todo, la amplia aceptación de la helioterapia terminó alterando el diseño original de muchos sanatorios. Uno de los más destacados fue el proyecto de ampliación de la clínica del doctor Bernhard en 1917 (imagen 9). Para ello, el arquitecto Nicolaus Hartmann propuso cinco niveles escalonados de habitaciones que ascendían entre dos torres de cantería. La influencia del Freilufthaus era evidente en su sección transversal, no solo por el uso de galerías en semi-ménsulas que arrancaban de la fachada, sino también por haber apostado decididamente por un sistema escalonado tal como reclamaba su promotor, el doctor Oskar Bernhard, en su libro Sonnenlicht behandlung in der Chirurgie de 1917. En cualquier caso, el estallido de la Primera Guerra Mundial condenó a este y a otros proyectos al ámbito de las publicaciones teóricas o de las obras no realizadas. Hubo que esperar hasta el período de entreguerras para que la sección escalonada del Freilufthaus se materializase, primero, en el contexto sanitario, para más tarde convertirse en un ejemplo paradigmático para la residencia colectiva urbana. Asimismo, aunque Richard Döcker planteó la generalización del Terrassenbau a toda una ciudad, el cambio de escala definitivo no se produjo hasta los años sesenta. Fue entonces cuando la versión más evolucionada del Freilufthaus dio forma a la mega-estructura, utilizando su sección escalonada como generatriz de la ciudad-paisaje.
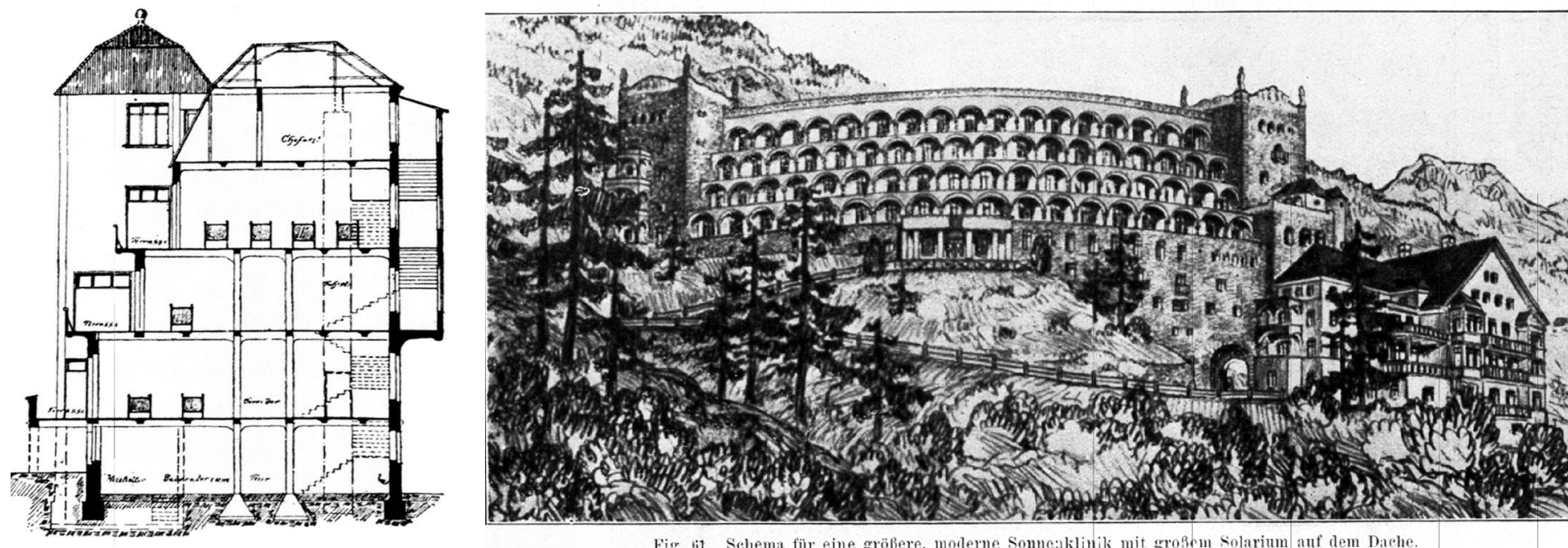

Fig. t1. Schema für eine größere, moderne Sonnc:aklinik mit großom Solarium auf dem Dache. 
CONCLUSIONES. A pesar de su vocación rupturista con los postulados del urbanismo moderno, la generación de arquitectos de la década de 1960 convergió con las ideas de algunas de las utopías propuestas por la vanguardia arquitectónica durante el período de entreguerras, al menos en lo que atañe a la necesidad de identificar arquitecturas que pudiesen dar forma a la ciudad mientras aseguraban un hábitat saludable para la residencia colectiva urbana. Los diseños de Henri Sauvage y Adolf Loos o de Chanéac y Justus Dahinden, entre otros, así lo demuestran. Los primeros, se centraron en el acceso al aire puro y al sol como fuente de bienestar, mientras los segundos mostraron la misma determinación, pero buscando la armonía ecológica con el paisaje.

Además, todos coincidieron en instrumentalizar la sección de terrazas escalonadas como recurso arquitectónico para alcanzar sus objetivos, aplicándolo primero sobre los modelos de habitación colectiva para después hacerlo extensible al resto de la ciudad. Así se reconoce en la literatura de la época, cuando arquitectos como Döcker promovieron la generalización del edificio-terraza o Terrasenbau en su libro Terrassen-typ (1929). Se trata de una ambivalencia que también estuvo presente en la década de 1960, cuando la sección escalonada modeló a las mega-estructuras como una geografía vertical habitable y que tuvo en las propuestas utópicas de Henri Borie (1865) a uno de sus precedentes formales más reconocibles.

No obstante, fueron los médicos los primeros en apostar por la arquitectura de terrazas como modelo para el hábitat colectivo. Para ello, tomaron como referente las soluciones aportadas por la arquitectura hospitalaria desde 1900, haciendo énfasis en los sanatorios escalonados. Así se extrae de las publicaciones de Julius Grober (1932) o del propio Richad Döcker, en las que se recopilan los ejemplos más paradigmáticos desarrollados hasta la fecha. Tal era el caso del Waiblingen County Hospital (1929), cuya sección transversal con un doble escalonamiento de terrazas en las fachadas norte y sur se utilizó como esquema estructural recurrente en gran parte de los proyectos de los años sesenta.

Junto a Waiblingen se publica el modelo teórico del Freilufthaus (1900), elaborado por el doctor David Sarason para adecuar la arquitectura de los sanatorios antituberculosos a la helioterapia. Aunque Sarason preconizó las posibilidades que ofrecía su sistema de terrazas escalonadas en 1913, no sería hasta 1960 que este recurso formal se generalizaría. La continuidad de estos modelos debe ser buscada en los destinos turísticos litorales: de las estaciones francesas de La Grande Motte, construida ex nihilo por Jean Balladur entre 1962 y 1968, al urbanismo en la ladera de Reñaca o Viña del Mar en Chile, en donde las terrazas satisfacen el deseo de aproximarse al paisaje a través de una arquitectura abierta al aire libre y al sol. $\mathbf{\Delta} \boldsymbol{\theta}$

\section{REFERENCIAS}

Banham, R., 1976. Megastructure. Urban futures of the recent past. Londres: Thames and Hudson. Bernhard, O., 1917. Sonnenlichtbehand/ung in der Chirurgie. Sttutgart: Ferdinand Enke.

Borie, H. J., 1865. Aérodômes: Essai sur un nouveau mode de maisons d' habitation. Paris: Morris. Cook. P. 1970. Experimental Architecture. Londres: Studio Vista.

Cook. P. 1967 Architecture: Action and plan. Londres' Studio Vista

Dahinden, J., 1971. Stadstrukturen für morgen. Stuttgart: Verlag Gerd Hatje

Döcker, R., 1929. Terrassen-typ: Krankenhaus, Erholungsheim, Hotel, Bürohaus, Einfamilienhaus, Sieg/ungshaus, Miethaus, und die Stadt. Sttutgart: Wedekind.

Dumont. M. J 1984. La fondation Rochschild et les premières habitations à bon marché de Paris: 1900-1925.

Paris: Ministère de l'Urbanisme, du Logement et des Transports.

Gescheit, H., 1929. Neuzeitliche Hote/s und Krankenhäuser; ausgeführte Bauten und Entwürfe. Berlin-

Charlottenburg: Ernst Pollak Verlag

Giedion, S., 1929. Befreites Wohnen. Licht, Luft, Öffnung. Zürich: Orell Füssli.

Grober, J., 1932. Das deutsche Krankenhaus: Handbuch für Bau, Einrichtung und Betrieb der Krankenanstalten. Jena: Gustav Fischer.

Guevrekian, G., 1930. Hôtels [et] sanatoria. París: S. De Bonadona.

Habraken, J., 1961. De dragers en de mensen. Amsterdam: Scheltema \& Holkema
Jiménez, E. y Vargas, I. C. 2017. "Hotels and sanatoria: the influence of tuberculosis on mass tourism architecture." Historia ciencia saude-Manguinhos, 24, 243-260.

Loos, A., 1928. "Grand-Hotel Babylon". ReD Revue Svazu Moderni kultury 'Devetsil', 10, 337. Maki. F. 1964. Investigations in Collective Form. St. Louis: Washington University Press.

McHale, J, 1969. The Future of the Future. Nueva York: George Braziller

McHale, J., 1967. "2000 +". AD The Architectural Design, 67: 89.

Nyilas, A. 2006. "On MIT Boston Harbor Project, 1959." Journal of Architecture and Planning, 608, 181-188.

Ragon, M., 1966. Les cités de l'avenir. Paris: Encyclopédie Planète.

Ragon, M., 1963. Ou vivrons-nous demain? Paris: Robert Laffont.

Safdie, M. y Wolin, J., 1974. For Everyone a Garden. Cambridge: The MIT Press

Sarason, D., 1913. Das Freilufthaus, Ein Neues Bausystem Für Krankenanstalten Und Wohngebäude: Prämiert

Mit Der Goldenen Medaille Vom Internationalen Tuberkulosekongress In Washington 1908. Munich: J. F.

Lehmann

Sauvage, H., s/f. Immeuble pyramidalà gradins "Métropolis", quai de Passy, Paris 8e: pers. d'ensemble. SIAF/ Cité de l'architecture et du patrimoine/Archives d'architecture du XXe siècle.

Disponible en: http://archiwebture.citechaillot.fr 